\title{
Kolonkarzinom: Gentest erlaubt genauere Prognoseeinschätzung
}

\author{
Der Gentest ColDx soll bessere \\ Aussagen zur Prognose von Patien- \\ ten mit Kolonkarzinom im Stadium II \\ ermöglichen. Nun wurden die \\ Ergebnisse validiert.
}

—ür Patienten mit einem Kolonkarzinom im Stadium II und hohem Risiko wird eine adjuvante Chemotherapie empfohlen. Doch Daten mehrerer Studien weisen darauf hin, dass die bisher verwendeten histologischen und klinischen Marker nur eingeschränkte prognostische Aussagekraft aufweisen. Es wurden bereits molekulare Marker identifiziert, die allerdings noch weiter evaluiert werden müssen, z. B. der Verlust der Heterozygotie und der p53-Mutationsstatus.

ColDx ist ein Mikroarray-Test, mit dem die Genexpression in pathologischen Gewebeproben untersucht werden kann. Es wurde bereits gezeigt, dass er prognostische Aussagen über rezidivfreie Zeit und Gesamtüberleben erlaubt.
Ziel der vorliegenden Studie war es, ColDx weiter zu validieren. Hierfür wurden Gewebeproben aus der PhaseIII-Studie C9581 der Alliance for Clinical Trials in Oncology (Alliance; ehemals Cancer and Leukemia Group B, CALGB) verwendet. In der Studie wurde Edrecolomab im Vergleich zu alleiniger Beobachtung bei 1.738 Patienten mit Kolonkarzinom im Stadium II ohne Hochrisikofaktoren untersucht. Dabei zeigte sich kein Überlebensvorteil unter Edrecolomab.

Die Gewebeproben von 393 Patienten wurden mit dem ColDx-Test analysiert. Anhand der Ergebnisse wurden die Patienten in Gruppen mit hohem bzw. niedrigem Risiko eingeteilt. $55 \%$ der $\mathrm{Pa}$ tienten wurden so als Hochrisiko-Patienten klassifiziert. Nach Berücksichtigung prognostischer Faktoren wie eines Defekts in der Mismatch-Reparatur (MMR) hatten Patienten mit hohem Risiko eine signifikant kürzere rezidivfreie
Zeit (Hazard Ratio [HR] 2,03; p < 0,01). Nach 5 Jahren betrug die Wahrscheinlichkeit für Rezidivfreiheit bei ihnen $82 \%$ im Vergleich zu $91 \%$ bei Patienten mit niedrigem Risiko. Beim Gesamtüberleben bestand ein nicht signifikanter Zusammenhang mit dem ColDx-Ergebnis (HR 1,74; $\mathrm{p}=0,06$ ).

Fazit: Der ColDx-Test war in einer Subkohorte der Studie C9581 bei Vorliegen von prognostischen Faktoren wie dem MMR-Status mit der rezidivfreien Zeit assoziiert. Damit könnte dieser Test zusammen mit den traditionellen klinischen Risikomarkern verwendet werden, um Aussagen zur Prognose der Patienten zu präzisieren.

Judith Neumaier

Niedzwiecki D et al. Association Between Results of a Gene Expression Signature Assay and Recurrence-Free Interval in Patients With Stage II Colon Cancer in Cancer and Leukemia Group B 9581 (Alliance). J Clin Oncol. 2016;34(25):304753.

\section{Kolorektalkarzinome: Engmaschig überwachen}

\begin{abstract}
Kurativ behandelte Patienten mit Kolon- und Rektumkarzinom sollten laut Leitlinien zur frühzeitigen Rezidiventdeckung engmaschig überwacht werden. Doch ist ein Vorteil für dieses Vorgehen nicht immer klar erkennbar.
\end{abstract}

$\mathrm{D}$ rei Viertel der Patienten mit Kolorektalkarzinom erhalten die Erstdiagnose in einem frühen Stadium und können kurativ behandelt werden. Eine anschließende engmaschige Überwachung soll das Langzeitüberleben verbessern. Ziel ist es, frühzeitig ein Rezidiv bei asymptomatischen Patienten zu entdecken, die dann nochmals eine Chance auf kurative Behandlung erhalten. Ob die eng getaktete Überwachung tatsächlich die Prognose verbessert, wurde in der GILDA-Studie überprüft. Einbezogen waren Patienten mit Adenokarzinom von Kolon oder Rektum im Dukes-Stadium B2-C, bei denen sich nach Erstlinientherapie mit Resektion und ggf. adjuvanter Chemoradiotherapie keine Erkrankung mehr nachweisen ließ. Insgesamt wurden
1.228 Patienten randomisiert 2 Überwachungsprogrammen zugewiesen, die sich vor allem in der Häufigkeit diagnostischer Bildgebung unterschieden.

Innerhalb der median 62 Monate Follow-up absolvierten mehr als $90 \%$ der Patienten das vorgesehene Nachbeobachtungsprogramm. Zum Zeitpunkt der 1. Analyse war bei 250 Patienten ein Rezidiv aufgetreten, 218 waren verstorben. Mithilfe der intensiven bildgebenden Überwachung wurden Rezidive wie erwartet deutlich früher erkannt, wodurch sich auch das krankheitsfreie Überleben um 5,9 Monate im Vergleich zur Minimalüberwachungs-Gruppe verlängerte. Dieser Vorteil verlor sich allerdings beim primären Zielkriterium Gesamtüberleben wieder. Auch hinsichtlich des 2. pri- mären Studienziels, der Veränderung der gesundheitsbezogenen Lebensqualität, ergaben sich keine Unterschiede zwischen beiden Überwachungsgruppen.

Die Ergebnisse wurden u. a. aufgrund zunächst fehlender finanzieller Unterstützung relativ spät publiziert, sodass eine Vergleichbarkeit zu heute behandelten Patienten nicht immer gegeben sei, diskutieren die Forscher. Dennoch sind die Resultate in Einklang mit anderen randomisierten Studien wie FACS und CEA Second Look, die ebenfalls keine Vorteile bei engmaschiger Überwachung und damit der Früherkennung eines Rezidivs erbrachten.

Fazit: Kurativ behandelte Patienten mit Kolon- oder Rektumkarzinom profitieren weder beim Gesamtüberleben noch der gesundheitsbezogenen Lebensqualität von einer engmaschigen bildgebenden Kontrolle.

Barbara Kreutzkamp

Rosati $G$ et al. A randomized trial of intensive versus minimal surveillance of patients with resected Dukes B2-C colorectal carcinoma. Ann Oncol. 2016;27(2):274-80. 